\title{
ALGORITMO BASEADO EM AFUNDAMENTOS DE TENSÃO PARA LOCALIZAÇÃO DE FALTAS EM ALIMENTADORES DE DISTRIBUIÇÃO DE ENERGIA ELÉTRICA
}

\author{
Rodrigo A. F. Pereira*
}

Luis G. W. da Silva ${ }^{\dagger}$

\author{
José R. S. Mantovani* \\ *Departamento de Engenharia Elétrica \\ Universidade Estadual Paulista - UNESP \\ Caixa Postal 31 \\ CEP 15385-000 - Ilha Solteira/SP \\ ${ }^{\dagger}$ Escola de Engenharia e Computação \\ Universidade Federal de Goiás- UFG
}

\section{RESUMO}

Neste trabalho é proposto um algoritmo baseado nas características de afundamentos de tensão para localização de faltas em alimentadores aéreos de distribuição de energia elétrica. Quando uma falta ocorre no alimentador, afundamentos de tensão propagam-se apresentando diferentes características para cada nó do alimentador. A utilização das características dos afundamentos de tensão garante a eficiência e a robustez do algoritmo, o qual fornece resultados adequados para a localização da falta. Um alimentador aéreo, real, trifásico a três fios, 13,8 kV, com 134 nós é utilizado para avaliar o algoritmo proposto. Os resultados dos testes mostram que o algoritmo fornece não somente o provável local da falta, mas também uma área contendo este local. Essas informações são extremamente úteis para as equipes de manutenção localizarem a falta e restabelecerem o fornecimento de energia elétrica.

PALAVRAS-CHAVE: Localização de faltas, afundamentos de tensão, distribuição de energia elétrica.

\section{ABSTRACT}

In this work, an algorithm based on the voltage sag characteristics for locating faults on overhead distribution feeders is proposed. When a fault occurs in the feeder, voltage sags propagate presenting different characteristics for each feeder node. The usage of voltage sags ensures the efficiency and robustness of the algorithm, which provides quite suitable results for fault location. An overhead, $13.8 \mathrm{kV}$, three-phase, three-wire, 134-node, real feeder is used in order to evaluate the algorithm. Test results show that the algorithm provides not only the likely faulty node, but also an area enclosing the faulty node is established. This information is extremely useful for maintenance crews to locate the fault and reestablish the power supplying.

KEYWORDS: Fault location, voltage sags, electric power distribution.

Artigo submetido em 10/07/2007

1a. Revisão em 22/12/2007

2a. Revisão em 02/04/2008

Aceito sob recomendação do Editor Associado

Prof. Carlos A. Castro 


\section{INTRODUÇÃO}

Faltas em alimentadores de distribuição causam atuação do sistema de proteção, interrompendo o fornecimento de energia elétrica aos consumidores. Geralmente, as faltas são ocasionadas por animais e árvores em contato com partes energizadas da rede de distribuição, severas condições de tempo, tais como raios, ventos, bem como tempo de vida útil e manutenção inadequada dos equipamentos. Normalmente, determinar o local ou a região de ocorrência de uma falta com precisão adequada é uma tarefa difícil de ser executada. Isso faz com que indicadores de continuidade do fornecimento de energia elétrica dependentes do tempo da interrupção, estabelecidos pela Resolução $\mathrm{n}^{o}$ 24/2000 (ANEEL, 2000), tais como DEC, DIC e DMIC, sofram degradação.

Uma das formas que as distribuidoras têm para minimizar a degradação dos indicadores, observando o padrão de continuidade, é investir em técnicas que proporcionam a localização de faltas nos alimentadores de maneira rápida e eficiente. Um procedimento muito usado pela maioria das distribuidoras de energia é um processo de localização de faltas baseado em tentativas e erros. Assim, através do conhecimento dos operadores quanto ao comportamento das redes de distribuição frente às contingências históricas ocorridas nestas redes, operações de abertura e fechamento de chaves são realizadas no alimentador para localizar a falta e isolar a região afetada. Porém, na maioria das vezes, esse processo é trabalhoso e resulta em elevados tempos de interrupção de energia. Após a implantação dos centros de atendimento aos clientes pelas distribuidoras de energia elétrica, o processo de tentativas e erros para a localização de faltas foi melhorado com informações baseadas em reclamações de clientes afetados com a interrupção do fornecimento de energia elétrica. Entretanto, mesmo com essas novas informações, o processo de localização de faltas ainda necessita ser otimizado.

Diante dessa necessidade e da rápida evolução da tecnologia e dos equipamentos para aquisição, processamento e transmissão de sinais elétricos, vários algoritmos e técnicas para localização de faltas em alimentadores de distribuição de energia elétrica têm sido propostos.

Lehtonen et alii (1991) apresentam um algoritmo baseado em fasores de frequiência fundamental das tensões e das correntes medidas no nó inicial do alimentador e no cálculo da impedância aparente para determinar o local da falta. El-Hami et alii (1992) propõem uma técnica para localização de faltas para alimentadores de distribuição baseada na detecção de componentes de altas frequências geradas pelas faltas. $\mathrm{O}$ local da falta é determinado através de processamento adequado dos sinais de alta frequência gerados pela falta na rede de distribuição. Girgis e Fallon (1992) e Girgis et alii (1993) propõem algoritmos baseados em fasores de freqüência fun- damental das tensões e das correntes medidas no nó inicial do alimentador e no cálculo da impedância aparente para determinar o local da falta. Jarventausta et alli (1994) apresentam uma metodologia baseada em informações de detectores de falta instalados ao longo do alimentador e tratamento das informações através de lógica fuzzy. Um algoritmo iterativo baseado em tensões e correntes superpostas é proposto por Aggarwal et alli (1997). Zhu et alli (1997) apresentam um algoritmo iterativo que calcula a distância da falta baseado em fasores de frequiência fundamental das tensões e correntes medidas no nó inicial do alimentador e em análises probabilisticas e diagnóstico de casamento de formas de ondas e carga rejeitada após a eliminação da falta. Das et alli (2000) propõem um algoritmo baseado em fasores de frequiência fundamental das tensões e das correntes medidas no nó inicial do alimentador e no cálculo da impedância aparente para determinar o local da falta. Uma técnica baseada na integração de informações de indicadores de faltas instalados ao longo do alimentador, cálculo da impedância aparente a partir dos fasores das tensões e das correntes medidos no nó inicial do alimentador e informações estatísticas de frequiência de faltas no alimentador é proposta por Lehtonen et alli (2001). Abur e Galijasevic (2002) propõem uma técnica de localização de faltas para sistemas de transmissão de energia elétrica que utiliza medições de tensões. Esta técnica é baseada no conceito de contornos de vulnerabilidade, os quais são usados para avaliar a possibilidade de afundamentos de tensão afetarem um dada área da rede de transmissão. Um algoritmo iterativo que calcula a distância da falta baseado em fasores de frequiência fundamental das tensões e correntes medidas no nó inicial do alimentador e em diagnóstico e análise de casamento de formas de ondas e carga rejeitada após a eliminação da falta é apresentado por Lee et alli (2004). Luo et alii (2004) propõem uma técnica de localização de faltas para sistemas de transmissão de energia elétrica que utiliza medições de tensões. O conceito básico desta técnica é a comparação dos fasores medidos durante a falta com fasores obtidos por simulações para a falta. Gohokar e Khedkar (2005) apresentam um algoritmo para localização de faltas em sistemas de distribuição automatizados que utiliza tensões e correntes medidas nos nós iniciais e finais de todas as seções de linha considerada. Um programa de análise de sistemas elétricos de potência em associação com métodos de busca em base de dados, técnicas de reconhecimento de padrões, além do uso da magnitude e do ângulo de fase de afundamentos de tensão medidos no nó inicial do alimentador, é proposto por Li et alii (2005). Senger et alli (2005) apresentam um sistema para localização de faltas que utiliza um algoritmo iterativo para calcular a distância da falta baseados em fasores de freqüência fundamental das tensões e correntes medidas no nó inicial do alimentador e em diagnóstico e análise de carga rejeitada após a eliminação da falta. Uma metodologia empregando medições de tensões e correntes em 
vários pontos do alimentador, teoria de grafos orientados e análise de circuitos elétricos é apresentada por Won et alli (2006). Pereira et alii (2006) apresentam uma técnica para localizar faltas fase-terra em alimentadores de distribuição que usa simulações de faltas e comparação de tensões medidas e simuladas para a condição de falta. Medições remotas devem estar disponíveis no alimentador. Esta técnica é de fácil aplicação para localizar faltas fase-terra, e sua eficiência e robustez é assegurada pelos resultados dos testes realizados. Porém a aplicação desta técnica não é tão simples para localizar outros tipos de faltas, como por exemplo, faltas trifásicas, devido à complexidade envolvida na modelagem para o cálculo das resistências de falta utilizadas nas simulações. Um modelo de programação binário irrestrito resolvido através de um algoritmo genético especializado para localização de seções em faltas em sistemas de energia elétrica é proposto por Leão et alli (2006). Um método baseado em redes de Petri é apresentado por Medeiros et alli (2007). Este método utiliza as informações disponibilizadas pelos relés ao sistema de supervisão e controle e é capaz de diagnosticar e localizar faltas em barras e em linhas. Embora sejam independentes da topologia da rede elétrica, os métodos propostos por Leão et alli (2006) e Medeiros et alli (2007) utilizam grande número de informações para localizar a seção em falta em um sistema de energia elétrica e não possuem habilidade para indicar o local de uma falta em um alimentador de distribuição radial e ramificado. São, portanto, adequados para diagnosticar e identificar seções e componentes em falta, como por exemplo, linhas de transmissão, disjuntores, barramentos e transformadores.

O algoritmo para localização de faltas proposto neste trabalho tem a habilidade de indicar o local ou a região da falta em um alimentador radial com vários ramais laterias e é baseado na comparação de tensões esparsamente medidas com tensões simuladas para a condição de falta. O algoritmo é adequado para localizar faltas em alimentadores aéreos de distribuição que não possuem sistemas de automação e controle implantados ou que têm sistemas de automação e controle rudimentares, independentemente do tipo de falta, pois nenhuma hipótese sobre a impedância de falta é necessária para a aplicação do algoritmo. Para a localização da falta, utilizam-se os fasores pré- e durante a falta das tensões e correntes medidas no nó inicial do alimentador, as magnitudes das tensões durante a falta medidas em alguns nós do alimentador, bem como uma base de dados contendo parâmetros, tais como a topologia do alimentador, as impedâncias das seções da linha e a potência nominal dos transformadores conectados ao alimentador. O algoritmo de localização de faltas é apresentado na seção 2. Para validar a eficiência e a robustez do algoritmo, resultados e análises de simulações realizadas para um alimentador de distribuição de $13,8 \mathrm{kV}$ e 134 nós, pertencente a um sistema real de distribuição de médio porte, são apresentados na seção 3. Por fim, conclusões são apresentadas na seção 4.

\section{ALGORITMO PROPOSTO}

A maioria das redes aéreas de distribuição apresenta topologia radial, várias seções, ramais laterais trifásicos, bifásicos ou monofásicos, cargas dos mais variados tipos, relação $X / R$ baixa e ausência de transposição. Desta forma, um algoritmo para localização de faltas deve ter capacidade de lidar com essas características, empregando técnicas apropriadas para análise de tais redes. Para satisfazer esses requisitos, o algoritmo proposto neste trabalho foi desenvolvido com base no algoritmo de fluxo de potência do tipo backward/forward sweep (Cheng e Shirmohammadi, 1995), o qual é adequado e eficiente para ser aplicado às redes de distribuição. O princípio básico para concepção do algoritmo é que, na presença de faltas, afundamentos de tensão propagam-se, apresentando características diferentes para cada ponto do alimentador. Isso possibilita o uso de grandezas fornecidas por um sistema de medições esparsas de tensões ao longo do alimentador para fornecer, com precisão adequada, a região ou até mesmo o local da falta. O diagrama de blocos do algoritmo é ilustrado na figura 1 .

Para a localização da falta, são necessárias informações armazenadas em banco de dados e grandezas elétricas registradas para a condições de pré-falta e durante a falta. A seguir, detalham-se os principais aspectos do algoritmo proposto.

\subsection{Grandezas elétricas requeridas}

\subsubsection{Fasores pré-falta das tensões e das corren- tes}

Os fasores pré-falta das tensões e das correntes devem ser registrados no nó inicial do alimentador em falta. Esses fasores são empregados no cálculo da potência complexa total demandada para a condição pré-falta. Tal potência é usada na estimativa do carregamento dos transformadores do alimentador, como descrito na seção 2.4 .

\subsubsection{Fasores durante a falta das tensões e das correntes}

Os fasores durante a falta das tensões e das correntes são registrados no nó inicial do alimentador em falta e são utilizados no algoritmo para realizar a localização da falta, como descrito nas seções 2.5 a 2.8 .

\subsubsection{Magnitude das tensões durante a falta}

Além dos fasores de pré- e durante a falta, o algoritmo utiliza as magnitudes dos afundamentos de tensão medidas durante 


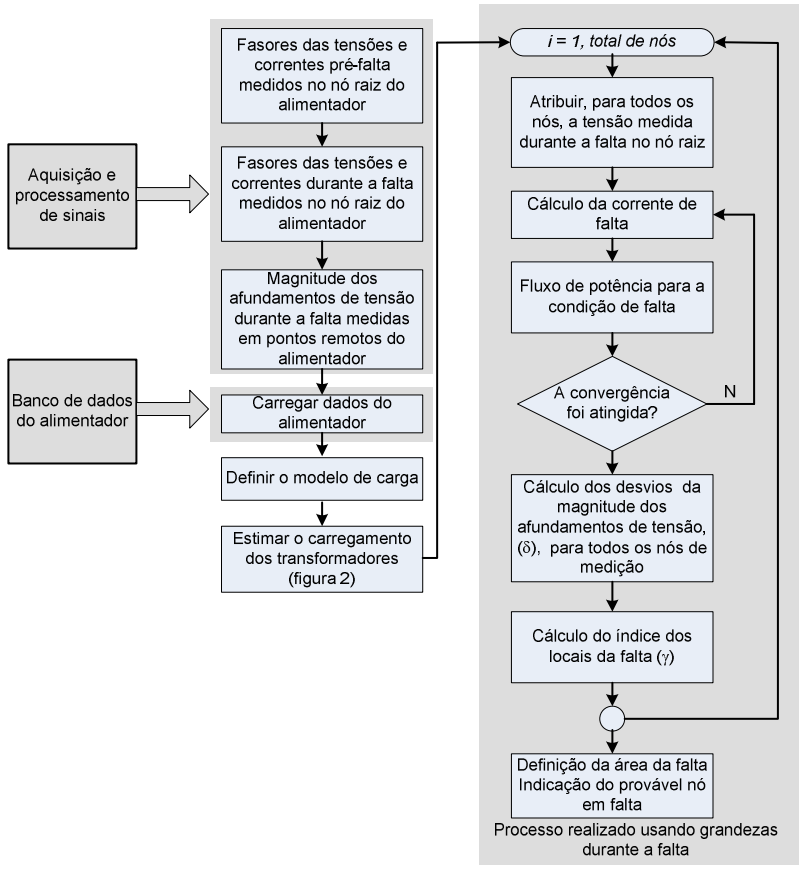

Figura 1: Diagrama de blocos do algoritmo proposto.

a falta em pontos esparsos ao longo do alimentador. Essas grandezas são utilizadas pelo algoritmo para fornecer a indicação do local da falta, como descrito na seção 2.7.

\subsection{Base de dados do alimentador}

A base de dados do alimentador é formada por informações topológicas, tais como comprimento das seções e locais de instalação de transformadores e dispositivos de proteção, e por informações elétricas, tais como impedâncias das seções, potência nominal dos transformadores e tensão nominal.

\subsection{Modelo de carga}

Geralmente as cargas presentes em um sistema de distribuição são especificadas pela potência complexa demandada por elas. Essas cargas podem ser trifásicas, bifásicas ou monofásicas. Neste trabalho, as cargas de potência constante, corrente constante ou impedância constante são modeladas como segue:

$$
\mathbf{S}=\mathbf{S}_{\text {nom }}\left(\frac{|\mathbf{V}|}{\left|\mathbf{V}_{\text {nom }}\right|}\right)^{q}
$$

sendo:

$\mathbf{S}_{n o m}$ : potência complexa nominal;
$\mathbf{V}_{\text {nom }}$ : tensão nominal da carga;

V: tensão aplicada aos terminais da carga.

Na equação (1), $q$ define o tipo da carga, sendo $q=0$ para potência constante, $q=1$ para corrente constante e $q=2$ para impedância constante.

Cargas que apresentam características mistas são modeladas através da combinação dos três tipos de cargas definidos na equação (1), a saber:

$$
\begin{array}{r}
S=\frac{\alpha}{100} \cdot \mathbf{S}_{\text {nom }}+\frac{\delta}{100} \cdot \mathbf{S}_{\text {nom }} \cdot\left(\frac{|\mathbf{V}|}{\left|\mathbf{V}_{\text {nom }}\right|}\right)+ \\
\frac{\beta}{100} \cdot \mathbf{S}_{\text {nom }} \cdot\left(\frac{|\mathbf{V}|}{\left|\mathbf{V}_{\text {nom }}\right|}\right)^{2}
\end{array}
$$

sendo:

$\alpha$ : porcentagem de carga de potência constante;

$\delta:$ porcentagem de carga de corrente constante;

$\beta$ : porcentagem de carga de impedância constante;

$\alpha+\beta+\delta=100,0$.

\subsection{Estimativa do carregamento dos transformadores}

Calcular o exato carregamento dos transformadores não é uma tarefa simples de ser realizada. Para propósitos de localização de faltas, o esforço despendido na estimativa ótima do carregamento dos transformadores pode não ser justificado, uma vez que os erros inseridos por carregamentos aproximados geralmente não comprometem a integridade do resultado final do algoritmo de localização de falta. Desta forma, para calcular o carregamento dos transformadores, é proposto um algoritmo iterativo baseado na seguinte equação:

$\mathbf{S}_{i}=\left(\lambda_{i} S_{i}^{N o m} \cdot \frac{S_{e s t}}{\sum_{k=1}^{n t} \lambda_{k} S_{k}^{N o m}}\right) \cdot\left[\cos \left(\varphi_{S E}\right)+j \operatorname{sen}\left(\varphi_{S E}\right)\right]$

sendo:

$S_{i}^{N o m}:$ Potência aparente do transformador;

$S_{\text {est }}$ : Potência aparente estimada; 


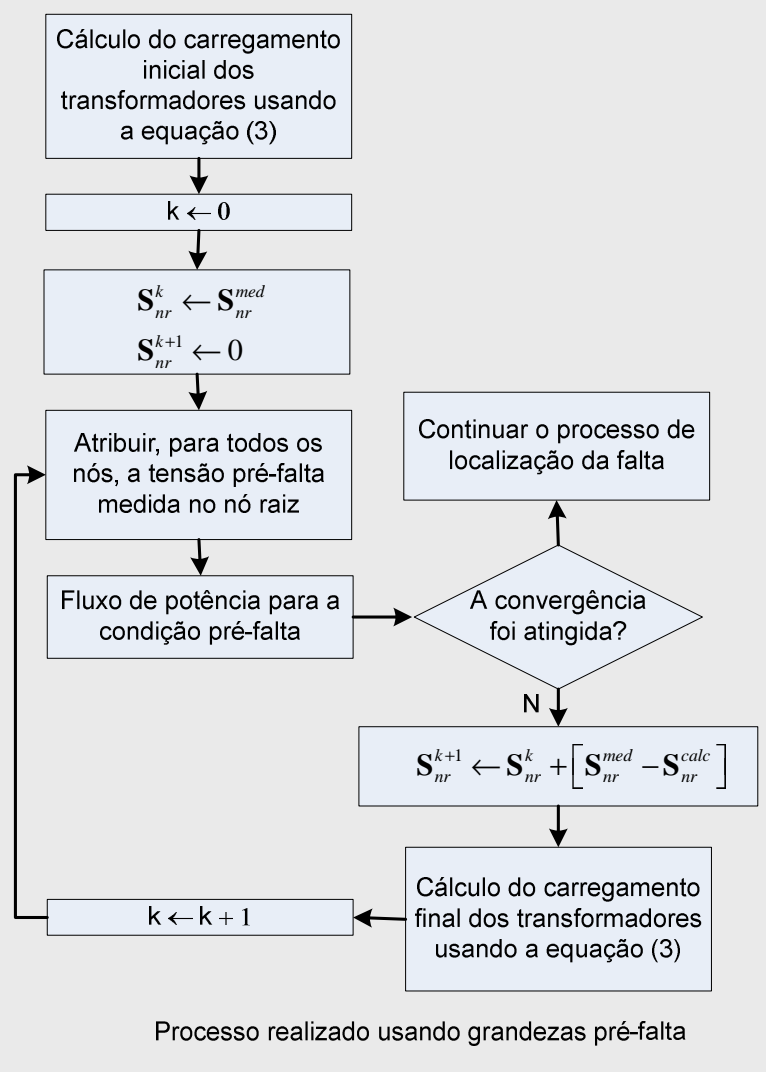

Figura 2: Procedimento para estimar o carregamento dos transformadores.

$\cos \left(\varphi_{S E}\right):$ Fator de potência medido no nó inicial;

$n t$ : Número total de transformadores instalados no alimentador;

$\lambda_{i}$ : Carregamento médio do transformador $i$.

A potência aparente medida no nó inicial do alimentador não pode ser utilizada diretamente na equação (3), pois a presença de perdas nessa potência faz com que ela seja diferente da potência aparente que deve ser usada na equação (3). Além disso, o algoritmo de localização de faltas deve reproduzir as mesmas condições de operação tanto em regime permanente quanto sob falta e, dependendo do modelo de carga adotado no algoritmo, essas condições podem não ser as mesmas.

O procedimento utilizado para estimar o carregamento dos transformadores é ilustrado na figura 2. O método de fluxo de potência empregado é o apresentado por Cheng e Shirmohammadi (1995) com as tensões do nó de referência iguais às tensões pré-falta medidas no nó inicial do alimentador.
As seguintes considerações são feitas com relação ao processo ilustrado na figura 2:

- A estimativa inicial do carregamento dos transformadores é feita através da equação (3) com $S_{e s t}=S_{n r}^{m e d}$;

- O fluxo de potência pré-falta sempre é iniciado com as tensões do nó de referência iguais às tensões pré-falta medidas no nó inicial do alimentador;

- A convergência é atingida quando $\left|\operatorname{Re}\left\{\mathbf{I}_{n r}^{\text {med }}\right\}-\operatorname{Re}\left\{\mathbf{I}_{n r}^{\text {calc }}\right\}\right| \leq \quad \leq \quad \mathrm{e}$ $\left|\operatorname{Im}\left\{\mathbf{I}_{n r}^{\text {med }}\right\}-\operatorname{Im}\left\{\mathbf{I}_{n r}^{\text {calc }}\right\}\right| \leq \varepsilon$, onde $\mathbf{I}_{n r}^{\text {med }}$ é a corrente medida no nó raiz, $\mathbf{I}_{n r}^{c a l c}$ é a corrente calculada para o nó raiz e $\varepsilon$ é a tolerância especificada;

- $\mathbf{S}_{n r}^{c a l c}$ é calculada em função dos fasores pré-falta das tensões e das correntes calculados pelo fluxo de potência;

- A estimativa final do carregamento dos transformadores é feita através da equação (3), com $S_{e s t}=S_{n r}^{k+1}$;

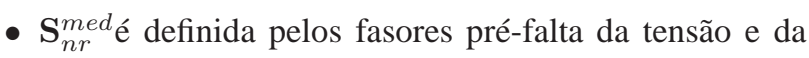
corrente medidos no nó inicial do alimentador.

\subsection{Fluxo de potência para a condição de falta}

No algoritmo de fluxo de potência apresentado por Cheng e Shirmohammadi (1995), o nó raiz é considerado como sendo o nó de referência, em que as magnitudes e os ângulos das tensões das fases são conhecidos. Para iniciar o algoritmo de fluxo de potência, consideram-se, ainda, as tensões iniciais para todos os nós do alimentador iguais às tensões do nó de referência. No algoritmo de localização de faltas, as tensões de referência são as tensões medidas no nó inicial do alimentador. O processo iterativo do algoritmo de fluxo de potência para alimentadores radiais consiste, basicamente, nos seguintes passos:

i. Calcular as correntes dos nós;

ii. Executar um passo backward para calcular as correntes nos ramos, isto é, inicia-se na seção de linha da última camada e move-se em direção ao nó raiz;

iii. Executar um passo forward para atualizar as tensões nodais, isto é, inicia-se na primeira camada e move-se em direção à última camada;

Uma vez que os três passos descritos acima foram executados dentro de uma mesma iteração, verifica-se a convergência. 


$$
\begin{gathered}
{\left[\begin{array}{c}
I_{j a} \\
I_{j b} \\
I_{j c}
\end{array}\right]=\left[\begin{array}{c}
\frac{\alpha}{100} \cdot\left(\frac{S_{j a}}{V_{j a}^{(n-1)}}\right)^{*}+\frac{\delta}{100} \cdot\left(\frac{\left(S_{j a}\right)^{*}}{\left|V_{n o m}\right|} \cdot \frac{V_{j a}^{(n-1)}}{\left|V_{j a}^{(n-1)}\right|}\right)+\frac{\beta}{100} \cdot\left(\frac{V_{j a}^{(n-1)}}{\left|V_{n o m}\right|^{2}} \cdot S_{j a}\right) \\
\frac{\alpha}{100} \cdot\left(\frac{S_{j b}}{V_{j b}^{(n-1)}}\right)^{*}+\frac{\delta}{100} \cdot\left(\frac{\left(S_{j b}\right)^{*}}{\left|V_{n o m}\right|} \cdot \frac{V_{j b}^{(n-1)}}{\left|V_{j b}^{(n-1)}\right|}\right)+\frac{\beta}{100} \cdot\left(\frac{V_{j b}^{(n-1)}}{\left|V_{n o m}\right|^{2}} \cdot S_{j b}\right) \\
\frac{\alpha}{100} \cdot\left(\frac{S_{j c}}{V_{j c}^{(n-1)}}\right)^{*}+\frac{\delta}{100} \cdot\left(\frac{\left(S_{j c}\right)^{*}}{\left|V_{n o m}\right|} \cdot \frac{V_{j c}^{(n-1)}}{\left|V_{j c}^{(n-1)}\right|}\right)+\frac{\beta}{100} \cdot\left(\frac{V_{j c}^{(n-1)}}{\left|V_{n o m}\right|^{2}} \cdot S_{j c}\right)
\end{array}\right]-\left[\begin{array}{c}
Y_{j a} \cdot V_{j a}^{(n-1)} \\
Y_{j b} \cdot V_{j b}^{(n-1)} \\
Y_{j c} \cdot V_{j c}^{(n-1)}
\end{array}\right]} \\
\Delta S_{j a}^{(n)}=V_{j a}^{(n)} \cdot\left(I_{j a}^{(n)}\right)^{*}-Y_{j a} \cdot\left|V_{j a}^{(n)}\right|^{2}-\left(\frac{\alpha \cdot S_{j a}}{100}+\frac{\delta \cdot S_{j a}}{100} \cdot \frac{\left|V_{j a}^{(n)}\right|}{\left|V_{n o m}\right|}+\frac{\beta \cdot S_{j a}}{100} \cdot\left(\frac{\left|V_{j a}^{(n)}\right|}{\left|V_{n o m}\right|}\right)^{2}\right) \\
\Delta S_{j b}^{(n)}=V_{j b}^{(n)} \cdot\left(I_{j b}^{(n)}\right)^{*}-Y_{j b} \cdot\left|V_{j b}^{(n)}\right|^{2}-\left(\frac{\alpha \cdot S_{j b}}{100}+\frac{\delta \cdot S_{j b}}{100} \cdot \frac{\left|V_{j b}^{(n)}\right|}{\left|V_{n o m}\right|}+\frac{\beta \cdot S_{j b}}{100} \cdot\left(\frac{\left|V_{j b}^{(n)}\right|}{\left|V_{n o m}\right|}\right)^{2}\right) \\
\Delta S_{j c}^{(n)}=V_{j c}^{(n)} \cdot\left(I_{j c}^{(n)}\right)^{*}-Y_{j c} \cdot\left|V_{j c}^{(n)}\right|^{2}-\left(\frac{\alpha \cdot S_{j c}}{100}+\frac{\delta \cdot S_{j c}}{100} \cdot \frac{\left|V_{j c}^{(n)}\right|}{\left|V_{n o m}\right|}+\frac{\beta \cdot S_{j c}}{100} \cdot\left(\frac{\left|V_{j c}^{(n)}\right|}{\left|V_{n o m}\right|}\right)^{2}\right)
\end{gathered}
$$

As equações para o cálculo das injeções de correntes e as equações para verificar a convergência, apresentadas por Cheng e Shirmohammadi (1995), foram definidas considerando somente cargas de potência constante. Desde que o algoritmo de localização de faltas deve considerar a modelagem adequada para as cargas, essas equações precisam ser redefinidas. Baseado nos modelos de carga definidos pela equação (2), o cálculo das injeções de correntes é definido através da equação (4), e a verificação da convergência é definida pela equação (5), sendo $S_{j}$ a potência complexa calculada usando a equação (3) como descrito na subseção 2.4.

\subsection{Estimativa da corrente de falta}

Para estimar a corrente de falta, considera-se que são conhecidas as tensões nodais e as correntes das cargas (fluxo de potência), assim como a corrente total medida no nó inicial do alimentador. Para ilustrar a estimativa da corrente de falta, seja o alimentador de distribuição, ilustrado na figura 3, com cargas nos nós $j$ e $k$ e uma falta trifásica à terra no nó $m$. A cada iteração do algoritmo, a corrente de falta é calculada através da equação (6).

$$
\mathbf{I}_{f}=\mathbf{I}_{n r}^{d f, \text { med }}-\sum_{i=1}^{n t} \mathbf{I}_{i}
$$

sendo:

$\mathbf{I}_{n r}^{d f, \text { med }}$ : Corrente medida no nó inicial do alimentador;

$\mathbf{I}_{i}$ : Corrente de carga do transformador $i$.

No algoritmo ora proposto, uma falta no alimentador de distribuição é tratada como sendo uma carga conectada no ali- mentador. A cada iteração, a corrente de falta calculada através da equação (6) é injetada no nó analisado, como ilustrado na figura 4. O uso de injeção de correntes faz com que nenhuma hipótese com relação à impedância de falta seja necessária, pois esta não aparece na modelagem da falta.

\subsection{Seleção do provável local da falta}

A seleção do provável nó em falta é efetuada considerando todos os nós analisados durante o processo de localização da falta. Para cada um dos nós analisados, determinam-se os desvios entre as magnitudes, calculadas e medidas, dos afundamentos de tensão durante a falta. Os desvios da magnitude dos afundamentos de tensão, para as fases envolvidas na falta, são definidos por:

$$
\begin{aligned}
& \delta_{k}^{i, j}=V_{k, \text { med }}^{i}-V_{k, \text { sim }}^{i, j} \\
& i=1, \ldots, n a \\
& j=1, \ldots, n m
\end{aligned}
$$

sendo:

$V_{k, \text { med }}^{i}$ : Magnitude do afundamento de tensão durante a falta, na fase $k$, medida no nó $i$;

$V_{k, \text { sim }}^{i, j}$ : Magnitude do afundamento de tensão para o nó $i$, na fase $k$, considerando uma falta simulada no nó $j$;

nm: Número de pontos de medições utilizados na localização de faltas;

na: Total de nós analisados.

A equação (7) fornece 3 desvios da magnitude dos afundamentos de tensão para faltas trifásicas, 2 desvios da magni- 
Nó raiz

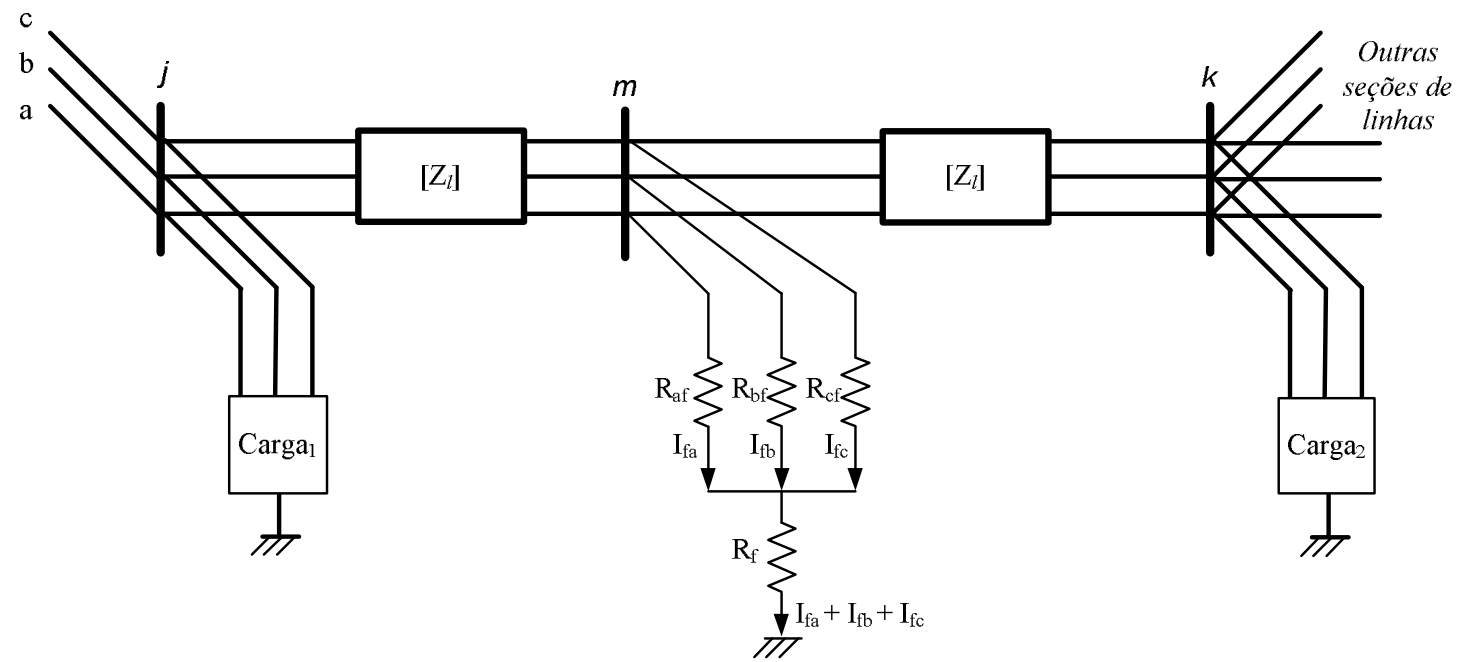

Figura 3: Representação de uma rede de distribuição com uma falta trifásica à terra.

Nó raiz

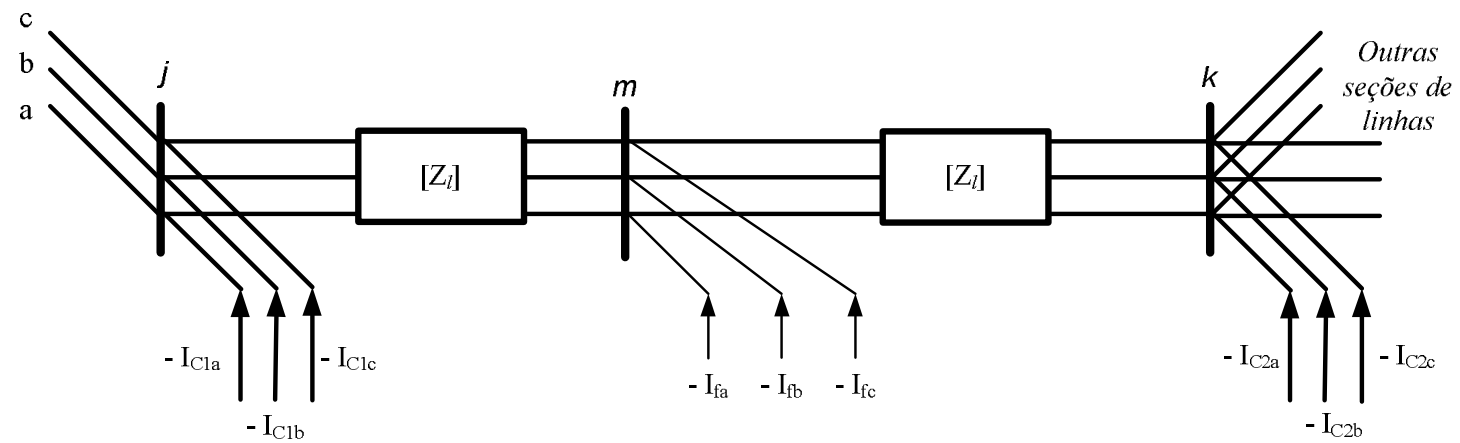

Figura 4: Injeção de corrente nos nós com cargas e no suposto ponto em falta.

tude dos afundamentos de tensão para faltas bifásicas e um desvio da magnitude dos afundamentos de tensão para faltas fase-terra. Considerando que a quantidade de desvios de tensão é proporcional ao tipo de falta, ao número de nós analisados e ao número de pontos de medição, é conveniente que, para cada nó analisado, os desvios de tensão sejam convertidos em um único índice. Assim, o índice do provável local da falta, $\gamma$, é definido por:

$$
\begin{aligned}
& \gamma_{i}=\min \left\{\max \left[\max \left(\delta_{k}^{i, j}\right)-\min \left(\delta_{k}^{i, j}\right)\right]\right\} \\
& i=1, \ldots, n a \\
& j=1, \ldots, n m
\end{aligned}
$$

sendo:
A região da falta é definida através da ordenação crescente dos $\gamma_{i}$. O provável nó em falta é definido como sendo aquele que apresenta o menor $\gamma$.

\subsection{Critério de Convergência}

A análise de cada um dos nós é feita de forma iterativa e a convergência é alcançada quando as condições impostas pela equação (9) são satisfeitas.

$$
\begin{array}{r}
\left|\operatorname{Re}\left\{\mathbf{I}_{n r}^{d f, \text { med }}\right\}-\operatorname{Re}\left\{\mathbf{I}_{n r}^{d f, \text { calc }}\right\}\right| \leq \varepsilon \\
\left|\operatorname{Im}\left\{\mathbf{I}_{n r}^{d f, \text { med }}\right\}-\operatorname{Im}\left\{\mathbf{I}_{n r}^{d f, \text { calc }}\right\}\right| \leq \varepsilon
\end{array}
$$

sendo: 


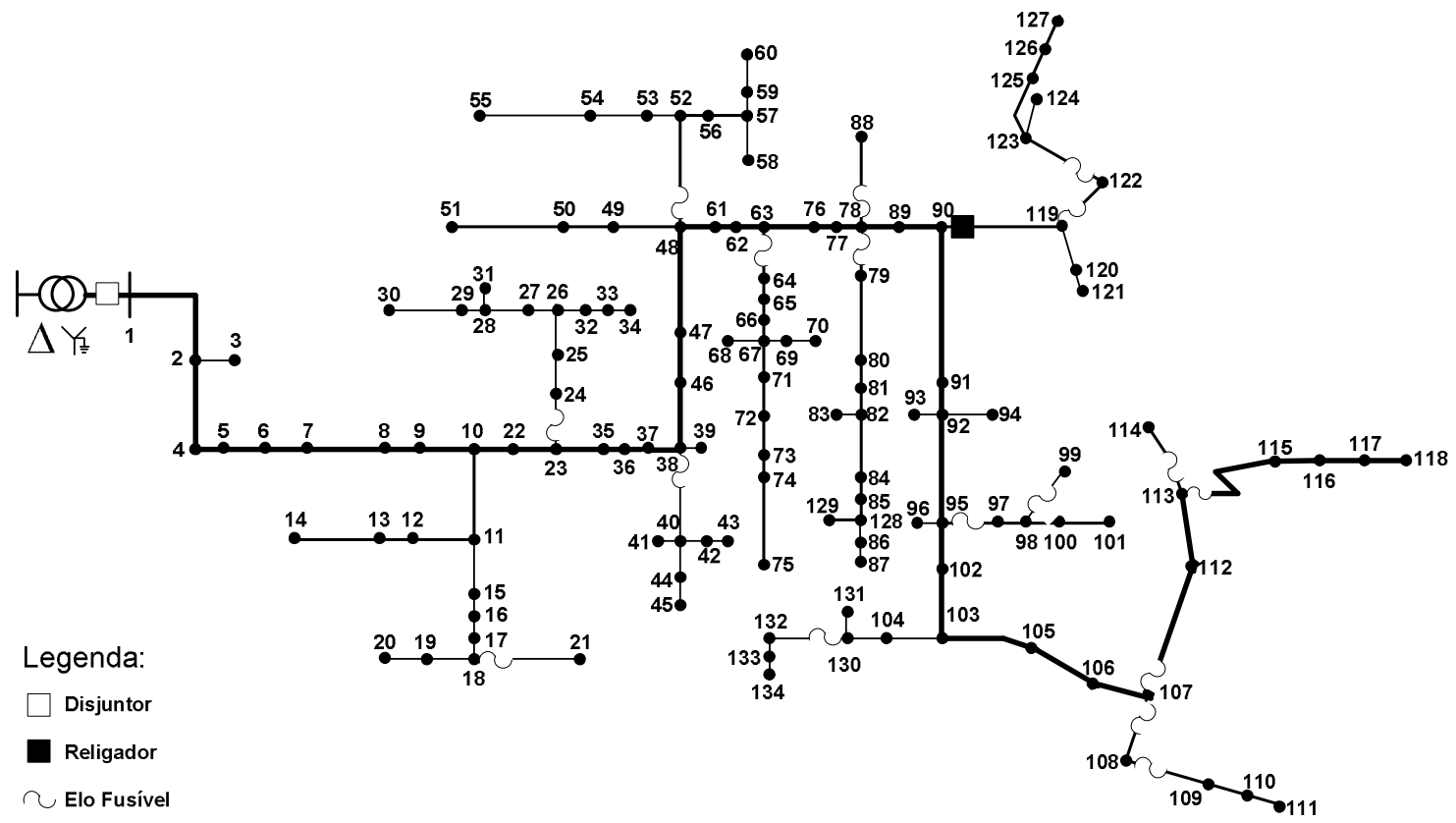

Figura 5: Alimentador real utilizado nas simulações.

$\mathbf{I}_{n r}^{d f, \text { med }}$ : corrente medida no nó inicial do alimentador;

$\mathbf{I}_{n r}^{d f, c a l c}$ : corrente calculada para o nó inicial do alimentador.

\section{RESULTADOS DAS SIMULAÇÕES}

Um alimentador real, aéreo, trifásico de 134 nós, $13,8 \mathrm{kV}$ e potência nominal instalada de 7,065 MVA, ilustrado na figura 5 , foi utilizado para avaliar o algoritmo proposto para localização de faltas. Esse alimentador contém vários ramais laterais, sendo que o nó 118 é o mais distante da subestação e está localizado a 4270,0 metros.

\subsection{Modelagem das linhas e das Cargas}

Tanto nas simulações no ATP (Comite Argentino de Usuarios del EMTP/ATP, 2002) quanto no algoritmo de localização de faltas, as linhas de distribuição foram consideradas como sendo não-transpostas, o modelo $R L$ série foi usado e as capacitâncias shunts foram desprezadas. Nas simulações no ATP, as cargas foram modeladas como impedâncias constantes; no algoritmo de localização de faltas as cargas foram modeladas como descrito na subseção 2.3.

\subsection{Simulação das faltas}

Para analisar a robustez e a precisão do algoritmo proposto, faltas foram simuladas em vários nós do alimentador. Para tanto, consideraram-se diferentes carregamentos, diferentes resistências de falta e diferentes tipos de faltas, sendo enfatizadas as faltas fase-terra. Os dados gerados pelas simulações foram usados como dados de entrada para o algoritmo de localização de faltas. A tabela 1 apresenta os parâmetros utilizados para algumas das simulações no ATP, sendo usado carregamento nominal para os transformadores.

Tabela 1: Parâmetros das simulações no ATP.

\begin{tabular}{|c|c|c|c|c|c|}
\hline \multirow{2}{*}{ Nó } & \multirow{2}{*}{$\begin{array}{c}\text { Tipo de } \\
\text { falta }\end{array}$} & \multicolumn{4}{|c|}{ Resistência de falta $(\Omega)$} \\
\hline & & $\mathbf{R}_{f}$ & $\boldsymbol{R}_{a f}$ & $\boldsymbol{R}_{b f}$ & $\boldsymbol{R}_{c f}$ \\
\hline $\begin{array}{c}15 ; 36 \\
40 ; 55 \\
105\end{array}$ & AN & $\begin{array}{c}1,0 ; 5,0 \\
10,0 ; 15,0 \\
20,0 ; 25,0\end{array}$ & - & - & - \\
\hline $15 ; 113$ & $\mathrm{BC}$ & - & - & 0,1 & 0,1 \\
\hline $16 ; 126$ & $\mathrm{ABCN}$ & 5,0 & 0,5 & 0,5 & 0,5 \\
\hline
\end{tabular}

\subsection{Medições remotas}

As localizações das faltas foram executadas considerando 4 medidores de tensão alocados nos nós 20, 81, 115 e 125 . A alocação dos pontos de medição foi feita de forma heurística. Essa heurística consiste basicamente em alocar os medidores em pontos distantes uns dos outros. Evita-se a alocação de mais de um medidor no ramal principal, pois é obtida uma maior diversidade das grandezas medidas com os medidores alocados em ramais laterais do que com medidores alocados 
no ramal principal. A alocação dos medidores de energia no alimentador impacta a precisão dos resultados do algoritmo, uma vez que, dependendo das características das cargas e da topologia do alimentador, a alocação de um medidor em um determinado nó pode melhorar ou degradar a precisão do algoritmo. Análises para alocação otimizada adotando funções objetivos que consideram somente o acerto ou não na localização da falta para algoritmos similares ao proposto foram abordadas em Pereira et alii (2004) e Pereira et alii (2006).

\subsection{Influência do modelo das cargas}

Como apresentado na subseção 3.1, nas simulações no ATP as cargas são modeladas como impedâncias constantes. De acordo com a equação (4), a variação da corrente de uma carga de potência constante é inversamente proporcional à variação da tensão aplicada na carga. A variação da corrente de uma carga de impedância constante é proporcional à variação da tensão aplicada à carga. Cargas de corrente constante somente sofrem alteração do ângulo de fase com a variação da tensão aplicada a seus terminais. Com o propósito de avaliar o desempenho do algoritmo de localização de faltas frente às variações das correntes das cargas quando nele se utiliza um modelo de carga diferente do modelo de carga (impedância constante) empregado nas simulações no ATP, seis diferentes combinações de cargas (CC1 a CC6) foram consideradas para as cargas conectadas no alimentador. Essas combinações são definidas de acordo com a equação (2), e os valores dos parâmetros $\alpha, \delta$ e $\beta$ são apresentados na tabela 2 .

Faltas foram aplicadas nos nós listados na tabela 1. Para localizar cada uma delas, o algoritmo de localização de faltas necessita analisar todos os nós de 2 a 134 da figura 5. Nesse processo de análise, o carregamento de cada transformador foi estimado como descrito na subseção 2.4 , considerando o carregamento médio igual à potência nominal, isto é, $\lambda=1,0$.

Tabela 2: Parâmetros das combinações de cargas utilizados no algoritmo.

\begin{tabular}{|c|c|c|c|}
\hline $\begin{array}{c}\text { Combinação de } \\
\text { Cargas }\end{array}$ & $\alpha$ & $\beta$ & $\delta$ \\
\hline CC1 & 0,0 & 100,0 & 0,0 \\
\hline CC2 & 0,0 & 75,0 & 25,0 \\
\hline CC3 & 0,0 & 50,0 & 50,0 \\
\hline CC4 & 0,0 & 25,0 & 75,0 \\
\hline CC5 & 0,0 & 0,0 & 100,0 \\
\hline CC6 & 15,0 & 60,0 & 25,0 \\
\hline
\end{tabular}

Para faltas fase-terra nos nós 55 e 105 não são verificados

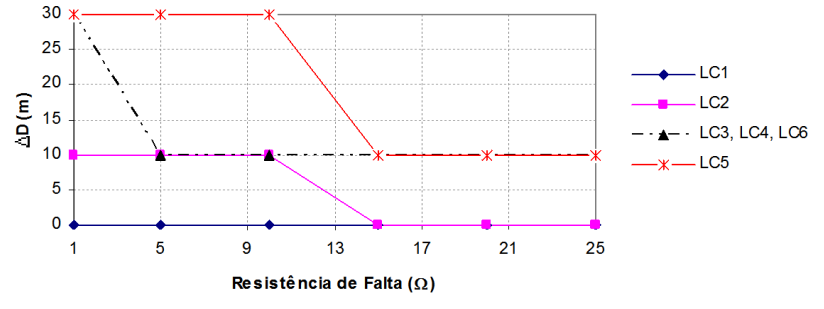

Figura 6: Distância entre o nó 15 e o local indicado pelo algoritmo.

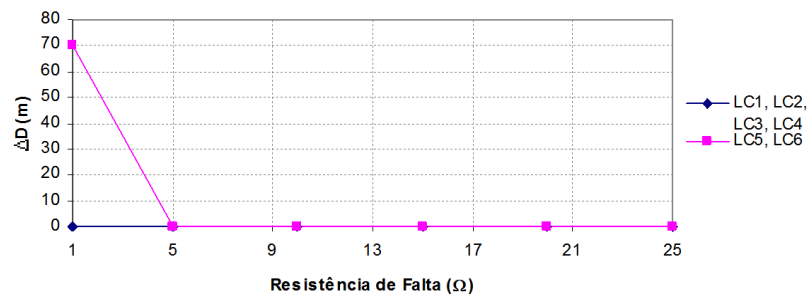

Figura 7: Distância entre o nó 36 e o local indicado pelo algoritmo.

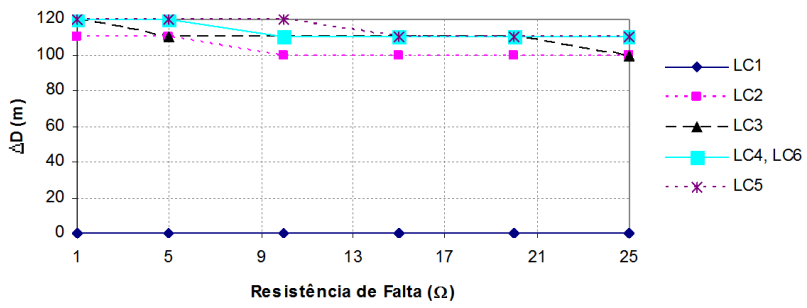

Figura 8: Distância entre o nó 40 e o local indicado pelo algoritmo.

erros na indicação do local da falta pelo algoritmo, isto é, os nós indicados pelo algoritmo coincidem com os nós onde foram simuladas as faltas. As figuras de 6 a 8 mostram as distâncias ( $\Delta$ D's) entre o nó em falta e o nó indicado pelo algoritmo para faltas fase-terra nos nós 15, 36 e 40.

Nas figuras 6 a 8 verifica-se que, para as faltas fase-terra, a maior distância entre o local da falta e o ponto indicado pelo algoritmo é 120 metros. Na maioria dos casos analisados, a distância encontra-se abaixo de 100 metros. Esses erros apresentados na distância entre o local da falta e o local indicado pelo algoritmo são aceitáveis, uma vez que o verdadeiro local da falta sempre está presente nas primeiras posições da lista dos prováveis nós em falta. Na figura 9 ilustra-se o resultado para a localização da falta fase-terra no nó $40 \mathrm{com}$ resistência de falta de $5,0 \Omega$, sendo utilizada no algoritmo a combinação de carga CC4.

Na figura 9, verifica-se que os $\gamma$ mantêm-se constantes para alguns nós. Assim, áreas contendo o possível local da falta 


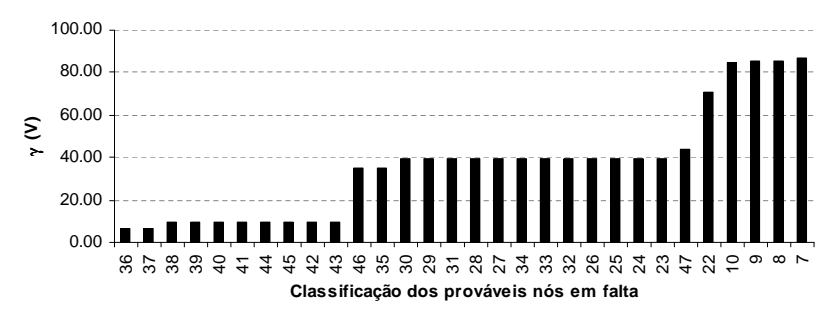

Figura 9: Trinta primeiros nós da lista dos prováveis nós em falta para a localização da falta fase-terra no nó 40 .

podem ser mapeadas. Essas áreas fornecem às equipes de manutenção subsídios suficientes para diminuir o tempo de localização da falta, de execução de reparos na rede e de restabelecimento do fornecimento de energia elétrica aos consumidores.

A figura 10 ilustra os $\Delta$ D's para a localização de faltas bifásicas nos nós 15 e 113 . O resultado da localização da falta bifásica no nó 113 , com emprego da combinação de carga CC4 no algoritmo, é mostrado figura 11.

Na figura 11, o nó em falta aparece na segunda posição da lista dos prováveis nós em falta, sendo que o nó da primeira posição da lista está a 110 metros de distância do nó 113 . Mesmo apresentando esse erro, a lista dos prováveis nós em falta fornecida pelo algoritmo é confiável.

Os $\Delta \mathrm{D}$ 's para a localização de faltas trifásicas à terra nos nós 16 e 126 são ilustrados na figura 12 . O resultado da locali-

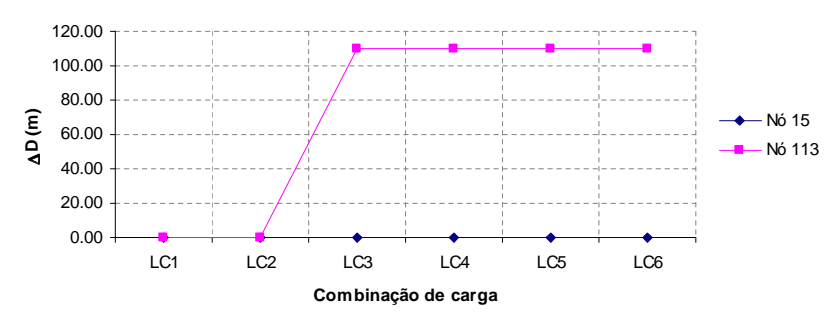

Figura 10: Distâncias entre o local da falta e o local indicado pelo algoritmo para faltas bifásicas nos nós 15 e 113 .

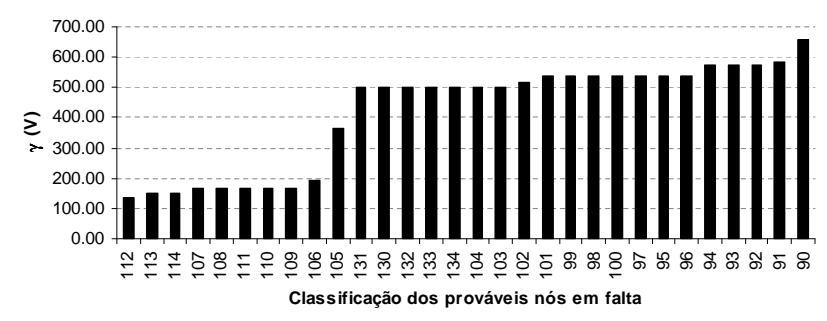

Figura 11: Trinta primeiros nós da lista dos prováveis nós em falta para a localização da falta bifásica no nó 113.

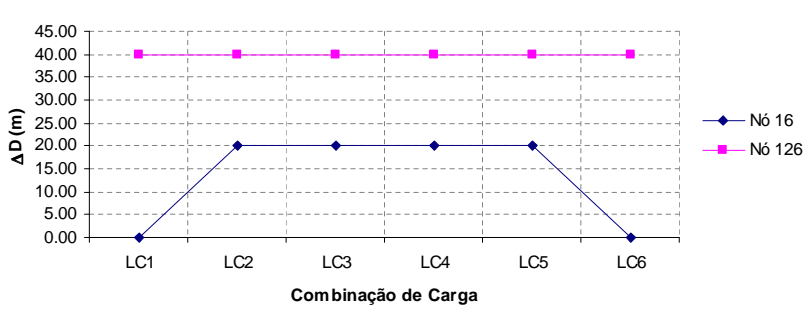

Figura 12: Distâncias entre o local da falta e o local indicado pelo algoritmo para faltas trifásicas à terra nos nós 16 e 126.

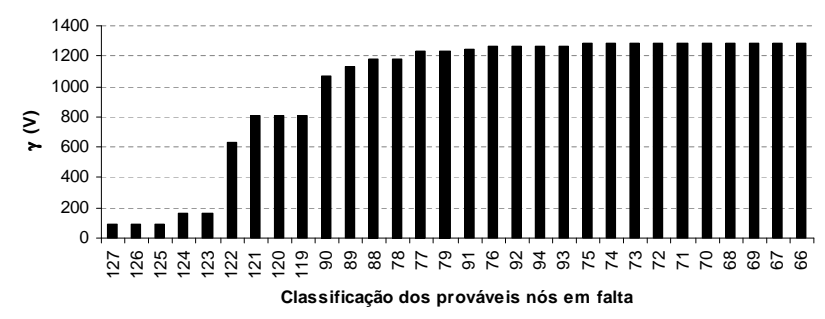

Figura 13: Trinta primeiros nós da lista dos prováveis nós em falta para a localização da falta trifásica à terra no nó 126.

zação da falta trifásica à terra no nó 126 , sendo utilizada a combinação de carga CC6 no algoritmo, é ilustrado na figura 13.

Na figura 13 o nó em falta aparece na segunda posição da lista dos prováveis nós em falta, sendo que o nó da primeira posição da lista está a 40 metros de distância do nó 126 . Novamente, verifica-se que a lista fornecida pelo algoritmo é confiável.

\subsection{Influência da incerteza do carrega- mento dos transformadores}

O algoritmo para localização de faltas estima o carregamento de cada transformador, baseado na potência complexa medida no nó inicial do alimentador, e da potência nominal de cada transformador instalado no alimentador, como apresentado na subseção 2.4. Desta forma, cada transformador terá o mesmo carregamento percentual com relação a sua potência nominal. Porém, devido às necessidades de cada consumidor, o carregamento dos transformadores tem comportamento estocástico. Isso faz com que os vários transformadores instalados no alimentador estejam com carregamentos diferentes quando a falta ocorre. Com o propósito de avaliar a influência da incerteza do carregamento dos transformadores, simulações no ATP foram executadas considerando um carregamento aleatório para cada transformador. Para essa finalidade, uma variável $\xi$ de distribuição normal, média um e desvio padrão $\sigma$, foi aleatoriamente selecionada. Assim, a potência complexa de cada transformador utilizada nas si- 
mulações no ATP é dada por:

$$
\mathbf{S}_{i}^{A T P}=\xi_{i} \cdot S_{i}^{N o m} \cdot(\cos \varphi+j \operatorname{sen} \varphi)
$$

sendo:

$\xi_{i}$ : variável aleatória de distribuição normal, média um e desvio padrão $\sigma$.

$S_{i}^{\text {Nom }}$ : Potência aparente nominal do i-ésimo transformador;

$\cos \varphi$ : fator de potência igual a $0,92$.

De acordo com a equação (10), para o i-ésimo transformador a diferença entre a potência aparente nominal e o carregamento utilizado nas simulações do ATP aumenta quando o desvio padrão $\sigma$ aumenta. Seis diferentes desvios padrões foram usados nas simulações de faltas nos nós 40 e 105 . A figura 14 ilustra a variável $\xi$ aleatoriamente selecionada da distribuição normal de média um para $\sigma=0,2$ e $\sigma=0,8$. Para faltas no nó 105, o algoritmo forneceu como resultado o mesmo nó em falta, isto é, o nó 105 . As distâncias $(\Delta \mathrm{D})$ entre o nó 40 e o nó encontrado pelo algoritmo são mostradas na figura 15. Diferentes resistências de faltas foram consideradas nas simulações no ATP. O carregamento utilizado no algoritmo foi estimado como descrito na subseção 2.4 e a combinação de carga utilizada foi a CC1.

A classificação crescente dos $\gamma$ para a localização de 2 faltas fase-terra da figura 15 é mostrada nas figuras 16 e 17 . Na figura 16, a falta foi simulada com resistência de falta igual a $25,0 \Omega$ e $\xi$ com média um e $\sigma=0,2$. Mesmo sendo o nó 40 classificado na sexta posição, o resultado fornecido pelo algoritmo é confiável. Isso porque o nó 43 , indicado na primeira posição, está a 60,0 metros do nó em falta. Ademais, todos os nós até a sexta posição estão situados na mesma região do alimentador e na zona de proteção de um elo-fusível.

Na figura 17, a falta foi simulada com resistência de falta igual a $1,0 \Omega$ e $\xi$ com média um e $\sigma=0,8$. Novamente o nó 40 é classificado na sexta posição, e mesmo que o nó 43

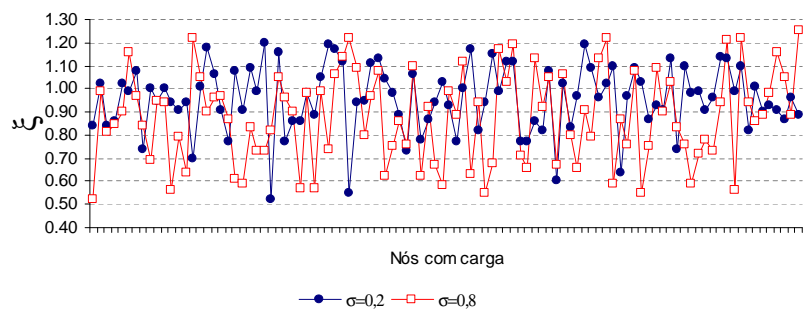

Figura 14: Variável aleatória $\xi$.

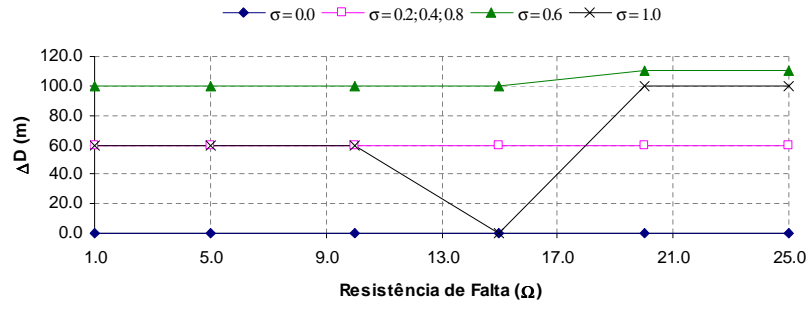

Figura 15: Distâncias entre o nó 40 e o nó encontrado pelo algoritmo para diferentes desvios padrões usados nas simulações.

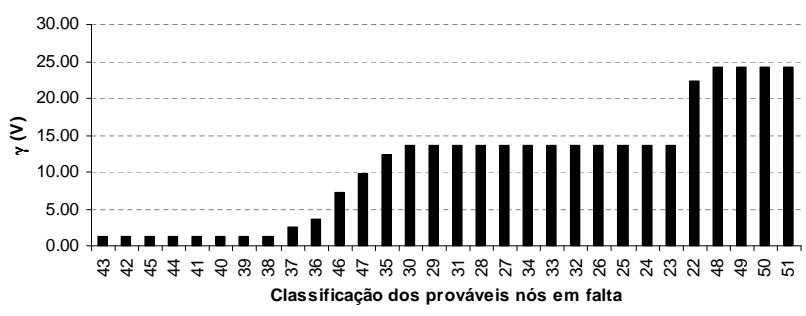

Figura 16: Classificação dos prováveis nós em falta para uma falta fase-terra simulada no nó 40 com resistência de falta igual a $25,0 \Omega$ e $\xi$ com média um e $\sigma=0,2$.

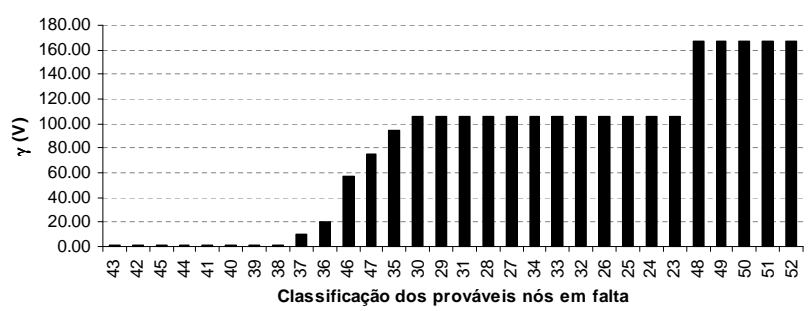

Figura 17: Classificação dos prováveis nós em falta para uma falta fase-terra simulada no nó 40 com resistência de falta igual a $1,0 \Omega$ e $\xi$ com média um e $\sigma=0,8$.

esteja a 100,0 metros do nó 40, esse resultado é de boa qualidade: os nove primeiros nós da lista pertencem a uma mesma posição geográfica do alimentador. Neste caso, a falta pode ser localizada facilmente usando a classificação da figura 17 e a informação de que esta falta não foi eliminada pelo disjuntor localizado no início do alimentador (o que ocasionaria a interrupção do fornecimento de energia a todos os clientes do alimentador).

\section{CONCLUSÕES}

Um algoritmo robusto e eficiente que utiliza medições esparsas de tensão para localização de faltas em alimentadores de distribuição de energia elétrica é aqui proposto. Esse algoritmo pode ser implantado com poucos investimentos adicionais pela maioria das empresas distribuidoras, as quais ne- 
cessitam, para tanto, de sistemas de aquisição de dados na subestação e em alguns pontos remotos do alimentador.

Os resultados obtidos com os testes executados mostram que o algoritmo desenvolvido é uma excelente ferramenta para auxiliar no processo de localização de faltas, principalmente quando as faltas estão ocultas na rede. Desta forma, o algoritmo proposto, aliado à sua simplicidade, é um excelente auxílio para indicar às equipes de manutenção qual direção deve ser tomada, minimizando, portanto, os tempos de busca do local do defeito, reconfiguração da rede, reparos e restabelecimento do fornecimento de energia elétrica. Todos os testes executados com o algoritmo proposto foram para alimentadores radiais e ramificados sem a presença de reguladores de tensão. Assim, novas pesquisas estão sendo desenvolvidas para avaliar o impacto da inclusão, na precisão e no desempenho do algoritmo proposto, de modelos disponíveis de reguladores de tensão para o método de fluxo de potência empregado pelo algoritmo.

\section{AGRADECIMENTOS}

Os autores agradecem a FEPISA (processo $\mathrm{n}^{\circ}$ 007/2005), CAPES (processo BEX0769/05-3) e CNPq o apoio recebido.

\section{REFERÊNCIAS}

Abur, A. and Galijasevic, Z. (2002). Fault location using voltage measurements. IEEE Transactions on Power Delivery, vol. $17, \mathrm{n}^{\circ} 2$, p.441 - 445 .

Aggarwal, R. K., Aslan, Y. and Johns, A. T. (1997). New concept in fault location for overhead distribution systems using superimposed components. IEE Proceedings $\mathrm{Ge}$ neration, Transmission and Distribution, vol. 144, $\mathrm{n}^{\circ} 3$, pp. $309-316$.

Aneel (2000). Resolução no 24. Agência Nacional de Energia Elétrica, Brasília, Brasil.

Comite Argentino de Usuarios del EMTP/ATP (2002). ATP Rulebook, Argentina.

Cheng, C. S. and Shirmohammadi, D. (1995). A three-phase power flow method for real-time distribution system analysis. IEEE Transactions on Power Systems, vol. 10, $\mathrm{n}^{\circ} 2$, pp. $671-679$.

Das, R., Sachdev, M. S. and Sidhu, T. S. (2000). A fault locator for radial subtransmission and distribution lines. IEEE Power Engineering Society Summer Meeting, pp. $443-448$.

El-Hami, M., Lai, L. L., Daruvala, D. J. and Johns, A. T. (1992). A new traveling-wave based scheme for fault detection on overhead power distribution feeders. Transactions on Power Delivery, vol. 7, $\mathrm{n}^{\circ} 4$, pp. 1825 1833.

Girgis, A. A. and Fallon, C. M. (1992). Fault location techniques for radial and loop transmission systems using digital fault recorded data. IEEE Transactions on Power Delivery, vol. 7, n ${ }^{o}$, pp. 1936 - 1945.

Girgis, A. A., Fallon, C. M. and Lubkeman, D. L. (1993). A fault location technique for rural distribution feeders. IEEE Transactions on Industry Applications, vol. 29, $\mathrm{n}^{o} 6$, pp. $1170-1175$.

Gohokar, V. N. and Khedkar, M. K. (2005). Faults locations in automated distribution system. Electric Power Systems Research, vol. 75, pp. $51-55$.

Jarventausta, P., Verho, P. and Partenen, J. (1994). Using fuzzy sets to model the uncertainty in the fault location process of distribution networks. IEEE Transactions on Power Delivery, vol. 9, n ${ }^{\circ}$ 2, pp. $954-960$.

Leão, F. B., Silva, L. G. W. e Mantovani, J. R. S. (2006). Localização de faltas em sistemas de energia elétrica através de um modelo de programação binária e algoritmo genético. XVI Congresso Brasileiro de Automática, pp. $631-636$.

Lee, S. J., Choi, M. S., Kang, S. H., Jin, B. G., Lee, D. S., Ahn, B. S., Yoon, N. S., Kim, H. Y. and Wee, S. B. (2004). An intelligent and efficient fault location and diagnosis scheme for radial distribution systems. IEEE Transactions on Power Delivery, vol. 19, $\mathrm{n}^{\circ} 2$, pp. 524 -532 .

Lehtonen, M., Pettissalo, S. and Etula, J. H. (1991). Calculational fault location for electrical distribution networks. IEEE Proceedings of the third International conference on Power System Monitoring and Control, pp. 38 - 43.

Lehtonen, M., Matsinen, A., Antila, E., Kuru, J., Vuorenpaa, P., Matinlassi, E. and Pettissalo, S. (2001). Automatic fault management in distribution networks. Proc of the 16th International Conference and Exhibition on Electricity Distribution, CIRED, vol. 3.

Li, H., Mokhar, A. S. and Jenkins, N. (2005). Automated fault location on distribution network using voltage sags measurements. Proc. of the 18th International conference and Exhibition on Electricity Distribution, CIRED.

Luo, S., Kezunovic, M. and Sevick, D. R. (2004). Locating faults in the transmission network using sparse field measurements, simulation data and genetic algorithm. Electric Power Systems Research, vol. 71, n ${ }^{\circ}$ 2, pp. 169 $-177$. 
Medeiros, E. B. de, Santos Fl, F. G., Colaço, A. L., Augusto Jr, M. F., Lourenço, T. G. M., Vieira, J. M. A., Sampaio, R. F., Barroso, G. C. e Leão, R. P. S. (2007). Uma Nova Abordagem para Diagnóstico de Faltas no Sistema Elétrico baseado em Redes de Petri Coloridas. XII ERIAC Encontro Regional Ibero-americano do CIGRÉ.

Pereira, R. A. F., Silva, L. G. W. and Mantovani, J. R. S. (2004). PMUs Optimized Allocation Using a Tabu Search Algorithm for Fault Location in Electric Power Distribution System. IEEE/PES Proceedings of the Latin America Transmission and Distribution Conference and Exposition.

Pereira, R. A. F., Silva, L. G. W., Kezunovic, M., and Mantovani, J. R. S. (2006). Location of single line-to-ground faults on distribution feeders using voltage measurements. IEEE/PES Proceedings of the Latin America Transmission and Distribution Conference and Exposition.

Pereira, R. A. F., Silva, L. G. W., Kezunovic, M. and Mantovani, J. R. S. (2006). Optimized Placement of Voltage Measurement Devices for Determining Location Of Single Line-To-Ground Faults On Overhead Electric Power Distribution Feeders. XVI Congresso Brasileiro de Automática.

Senger, E. C., Manassero Jr., G., Goldemberg, C., and Pellini, E. L. (2005). Automated fault location system for primary distribution networks. IEEE Transactions on Power Delivery, vol. 20, $\mathrm{n}^{\circ}$ 2, pp. $1332-1340$.

Won, D. J., Chung, I. Y., Kim, J. M., Moon, S. I., Seo, J. C. and Choe, J. W. (2006). A new algorithm to locate power-quality event source with improved realization of distributed monitoring scheme. IEEE Transactions on Power Delivery, vol. 21, no 3, pp. 1641 - 1674.

Zhu, J., Lubkeman, D. L., and Girgis, A. A. (1997). Automated fault location and diagnosis on electric power distribution feeders. IEEE Transactions on Power Delivery, vol. $12, \mathrm{n}^{\circ} 2$, pp. $801-809$. 